\title{
Programmable Control in Extracellular Matrix-mimicking Polymer Hydrogels
}

\author{
Kevin S. Hof and Maartje M. C. Bastings*
}

\begin{abstract}
The extracellular matrix (ECM) and cells have a reciprocal relationship, one shapes the other and vice versa. One of the main challenges of synthetic material systems for developmental cell culturing, organoid and stem cell work includes the implementation of this reciprocal nature. The largest hurdle to achieve true cellinstructive materials in biomaterials engineering is a lack of spatial and temporal control over material properties and the display of bioactive signals compared to the natural cell environment. ECM-mimicking hydrogels have been developed using a wide range of polymers, assembly and cross-linking strategies. While our synthetic toolbox is larger than nature, often our systems underperform when compared to ECM systems with natural components like Matrigel. Material properties and three-dimensional structure ill-represent the three-dimensional ECM reciprocal nature and ligand presentation is an oversimplified version of the complexity found in nature. We hypothesize that the lack of programmable control in properties and ligand presentation forms the basis of this mismatch in performance and analyze the presence of control in current state of the art ECM-mimicking systems based on covalent, supramolecular and recombinant polymers. We conclude that through combining the dynamics of supramolecular materials, robustness from covalent systems and the programmable spatial control of bio-activation in recombinant ECM materials, the optimal synthetic artificial ECM could be assembled.
\end{abstract}

Keywords: Bioactive materials · Cell-material interface $\cdot$ Extracellular matrix $\cdot$ Polymer hydrogels $\cdot$ Synthetic cell niches

\section{Introduction}

All biological materials are assembled from a small library of building blocks programmed by DNA. The simple four-base code found in our genetic material is translated into architectures that possess a wide range of materials properties and showcase complex dynamic robustness. Mimicking this level of programmable control in synthetic materials is challenging even with modern developments in chemistry and the availability of a larger engineering toolbox. When aiming to develop biomaterials that display the same degree of sequence and property control that nature offers, programmable control of polymerization and bio-activation is required.

${ }^{\star}$ Correspondence: Prof. Dr. M. M. C. Bastings Ecole Polytechnique Fédérale de Lausanne (EPFL) Institute of Materials, Programmable Biomaterials Laboratory

EPFL - STI - IMX - PBL, MXC 340, Station 12

$\mathrm{CH}-1015$ Lausanne

E-mail: Maartje.Bastings@epfl.ch
Cells rely on their extracellular matrix (ECM) which consists of several glycoproteins like collagen, fibronectin and laminin, ${ }^{[1]}$ to direct proliferation, adhesion and migration. ${ }^{[2-4]}$ Combining these proteins, the natural ECM has incredibly versatile functionality resulting in specific niche stiffness and spatio-temporal control of bioactive cues. ${ }^{[1,5,6]}$ Biomaterials that mimic the ECM have an influence on cell behavior through their stiffness and elasticity, reported as Young's modulus $(E)^{[7,8]}$ and robustness, e.g. degradability and adaptivity. ${ }^{[9]}$ Apart from the physical properties, biomaterials require bio-functionalization to imitate key properties of the natural ECM necessary for cell-adhesion and proliferation. Ligands mimicking natural ECM motifs are often included in biomaterials; $[10]$ however, control in spacing and orientation is limited by polymerization and chemical conjugation techniques.

Matrigel[11] is currently the gold standard in artificial ECM materials. While ill-defined from an engineering standpoint, it offers a well-defined and highly functional matrix from the cell's perspective. However, the carcinoma origin restricts future therapeutic translation. Many synthetic efforts have been undertaken to mimic the programmable natural matrix, ${ }^{[12]}$ though often without the desired cellular outcome (Fig.1). Introducing programmability in biomaterial design seems to be a parameter that is generally lacking and might be a reason why many attempts in ECM-mimicking hydrogels fail to ac- curately interact with the living subjects. In this review, we analyze state-of-the-art ECM-mimicking materials and search for programmable control within these systems. Through careful comparison of synthesis, material properties, bio-functionalization and cell interaction, we extract design parameters that aim to improve our understanding and control of the cell-material interplay to enable future advances in artificial ECM engineering.

\section{ECM-mimicking Materials}

The most prominent materials property of an ECM-mimicking hydrogel is matrix stiffness. Only materials with a specific stiffness are able to propagate and direct mechanosensitive differentiation by guiding nuclear stiffness and expression patterns. ${ }^{[13]}$ Tissue function is directly correlated to mechanical properties, and every specific tissue niche shows a defined range in stiffness: for example, neurons have a stiffness $E$ of $0.05-0.3 \mathrm{kPa},{ }^{[14]}$ versus $12 \mathrm{kPa}$ in muscles, ${ }^{[15]}$ and $20-110 \mathrm{kPa}$ in bone. ${ }^{[16]}$ Besides stiffness, the level of stable and controlled adaptive remodeling, e.g. dynamic robustness, influences cell development. Natural ECMs undergo continuous remodeling by developing cells and growing tissues, while retaining their structural integrity. ${ }^{[17]}$ Finally, charge and hydrophilicity determine adhesion or repulsion and influence cell fate. ${ }^{[18]}$ All these properties are optimized in the natural 
Artificial ECM mimicking materials without programmable control:

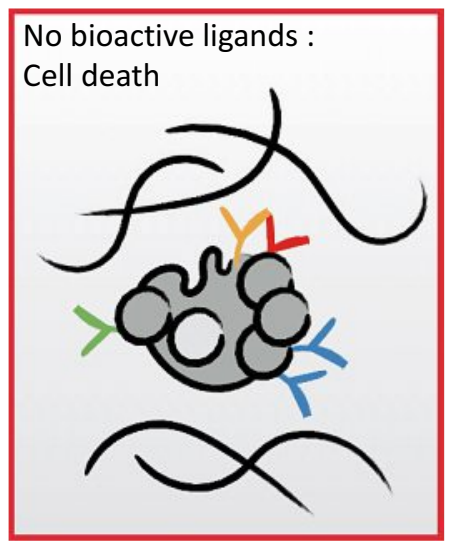

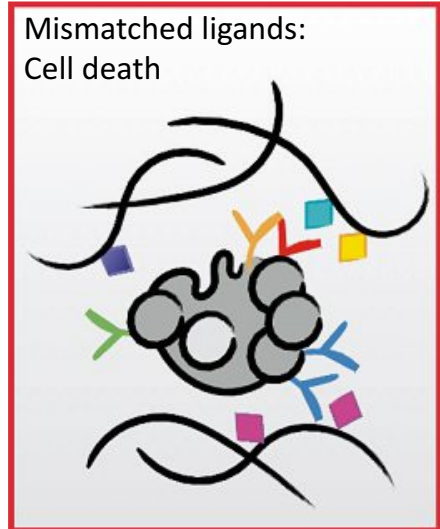

Nature:

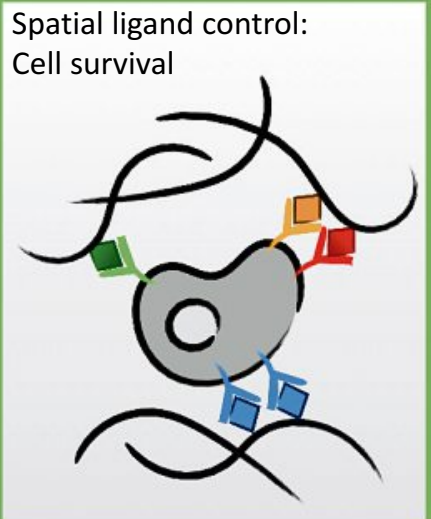

Fig. 1. Schematic representation of the engineering challenges in biomaterials; only with a perfect spatial control of bioactive ligand presentation, cells receive the necessary cues for proliferation and differentiation. When the material lacks bio-active cues (anti-fouling), if the cues are present but incorrectly spatially positioned, or when non-matching bio-actives are displayed, cells will not be able to interact with the material and experience cell death.

ECM but need to be controlled in the design of synthetic analogues. Bulk constituents of ECM hydrogels can be natural (e.g. collagen, fibronectin, Matrigel), bioactive polymers (e.g. alginate, hyaluronic acid, heparin, etc.), synthetic polymers (e.g. PEG, acrylate, acrylamide, PLGA, etc.), or a combination thereof. In order to explore current day progress towards a welldefined and programmable nature in ECM mimicking hydrogels, we will categorize the employed materials based on type of cross-linking.

\subsection{Programmable Material Properties in Covalent Polymer Hydrogels}

A plethora of different types of hydrogels exist that are cross-linked through various chemical mechanisms and built from constituents with a wide range of inherent properties. Covalent polymer hydrogels are chemically cross-linked and can be covalently bio-functionalized. Often these gels have one main constituent and therefore a controlled Young's modulus defined by the polymer chain length and crosslinking density. The range in stiffness of synthetic hydrogels developed to mimic the ECM is enormous, spanning five orders of magnitude from $0.3 \mathrm{kPa}^{[19]}$ to $8 \mathrm{MPa}$. ${ }^{[20]}$ In what follows, a selection of covalent polymer hydrogels showcasing state of the art systems, void hydrogels, photo-tunable hydrogels, matrix metalloproteinase (MMP) degradable hydrogels, hyaluronic acid-gelatin hydrogels, as well as the first synthetic system performing comparably to Matrigel will be reviewed and analyzed for their programmability.

Through incorporation of quickly degrading gel beads into a stable bulk hydrogel, voids can be formed that enable niche formation. Void hydrogels are assembled through calcium sulphate crosslinking of RGD-modified alginate and unmodified or slightly irradiated alginate (to reduce the molecular weight). To create 'voids', high guluronic acid (GA)-content alginates were partly oxidized into small gel beads (which rapidly degrade in situ) and added to the alginate material. Irradiation of the unmodified slowly degrading bulk gel resulted in a wide range of stiffness, spanning from 5-110 $\mathrm{kPa} .{ }^{[21]}$ The GAalginates create a temporal change in porosity making this material robust yet dynamic, as degradation is location-specific and tunable by the spread of GA beads. The use of alginate as the main backbone imposes a negative charge on the bulk architecture. Programmability in this system results from irradiation and oxidation of the bulk gel and GA-alginate gel respectively, allowing for independently creating gels with a different stiffness, pores, and degradation rates.

Photo-tunable hydrogels depend on external light to modulate their stiffness by degradation. The stiffness of photo-tunable hydrogels can thus be modulated by the exposure to irradiation, which provides a moderate programmable control of properties. A di-photodegradable acrylate PEG cross-linker allows for a directly tunable stiffness, decreasing from $10 \mathrm{kPa}$ to $2 \mathrm{kPa}$ in a dose-dependent fashion. ${ }^{\text {[9] }}$ Because of the use of a PEG backbone, no charge is present and the resulting architectures are anti-fouling.

Recently, the Lutolf group developed the first synthetic matrix rivalling the Matrigel standard.[19] In their approach, PEG polymers were enriched with fibronectin, laminin-111, collagen IV, hyaluronic acid, and perlecan to cre- ate a functional Matrigel-like hydrogel. Hydrogels in a stiffness range of 0.3-1.7 $\mathrm{kPa}$ and degradable PEG-alginate gels were produced. Dynamics are introduced through ester-based hydrolysis of the PEG hydrogel and by using alginate lyase for PEG-alginate degradation. Depending on the exact choice of the employed components, the polarity of the material can be altered. The programmability of degradation, through ester-based hydrolysis, is at the same level of matrix metalloproteinase (MMP)-degradable hydrogels and, while their synthetic matrix shows tremendous potential, due to its heterogeneous nature of degradable components, the programmability remains a challenge.

Hyaluronic acid-gelatin hydrogels ${ }^{[22]}$ with disulfide cross-links can be modulated through reduction with dithiothreitol (DTT) and enzymatically through collagenase, hyaluronidase, or incorporation of MMP degradable motifs. The stiffness of acrylated hyaluronic acid gels with MMP motifs ranged from $2.5-30 \mathrm{kPa}^{[23]}$ and in later work expanded to $4.4-91.6 \mathrm{kPa} .{ }^{24]}$ Due to the hyaluronic acid base, these materials are hydrophilic and anionic. The extensive biodegradability and addition of MMP provides a higher degree of control of degradation than found in Lutolf's matrix, however, they are less controlled than photo-tunable and void hydrogel systems.

Poly $(N$-isopropylacrylamide $)$ (PNIPAAm) is one of the most studied thermosensitive hydrogels and displays a lower critical solution temperature (LCST) at $32{ }^{\circ} \mathrm{C}$, which introduces unique material properties. At low temperatures, the material behaves like a liquid, is hydrophilic, and can be easily processed. When heated above the LCST, the polymer collapses to a dehydrated, hydrophobic state, los- 
ing $90 \%$ of its volume. When cross-linked with acrylamides, a soft thermoresponsive hydrogel network is formed. To use this as a cell-supportive biomaterial and obtain material properties in the biologically relevant range $(0.5-10 \mathrm{kPa})$, pNIPAAm needs to be copolymerized with biodegradable and hydrophilic polymers like poly(amidoamine), PEG and pNIPAAmPEG/alginate mixtures. ${ }^{[25]}$ In the PEGcopolymer composition, the hydrogel is commercially available under the name of Mebiol Gel.[26] Control of properties in pNIPAAM itself is limited; system properties are defined and adjusted by the choice of copolymers or mixed-in polymers.

\subsection{Programmable Material Properties in Supramolecular Architectures}

The thermodynamic, kinetic, and mechanical properties of polymers found in biology are the result of supramolecular interactions. Matching the sophistication of supramolecular polymerization in nature is still a grand challenge in synthetic polymer engineering. Synthetic polymer systems can contain biomolecular building blocks (e.g. peptide chains, proteins, biological macrocycles, and DNA) and utilize the intermolecular binding motifs in their supramolecular polymerization. Due to the supramolecular nature of the cross-links and bio-functionalization, hydrogels can possess inherent dynamics, exemplified by tunability through $\mathrm{pH}^{[27]}$ and temperature. ${ }^{[28]}$ The Young's modulus reported for supramolecular ECM mimics spans six orders of magnitude from $0.1 \mathrm{kPa}^{[29]}$ to $49 \mathrm{MPa} .^{[30]}$ Synthetic supramolecular polymers have classically been subdivided according to varying criteria, such as the physical nature of the intermolecular interactions, the type of monomer building blocks, or the thermodynamics of polymerization. ${ }^{[31]}$ Here, we focus on three ECM-mimicking materials systems based on supramolecular polymers that rely on a mechanistically different primary binding motif that governs their formation: (i) hydrogen-bonding motifs, (ii) peptides, and peptide amphiphiles, and (iii) systems based on DNA (self-assembled through a combination of hydrogen-bonds, $\pi-\pi$ stacking, and hydrophobic interactions). ${ }^{[32]}$

When hydrogen-bonding units are transferred to aqueous medium, the solvent competes with the hydrogen-bonding array of the synthetic molecules, significantly decreasing the strength and stability of this interaction. To achieve robust supramolecular polymerization in an aqueous environment, the hydrogen-bonding motif needs to be surrounded by a hydrophobic microenvironment, which shields it from water. ${ }^{[33]}$ In the Meijer laboratory, hydrogels using the quadruple hydrogenbonding 2-ureido-4[1H]-pyrimidinone (UPy) unit ${ }^{[34]}$ were generated by end-functionalization of the UPy with polyethylene glycol (PEG), spaced and shielded by a hydrophobic alkyl pocket and decorated with a urea motif primed for lateral hydrogen bonding. ${ }^{[35]}$ Mechanical properties range from $3 \mathrm{kPa}^{[35]}$ to $22 \mathrm{kPa}^{[36]}$ and can be tuned by length of alkyl spacer and the molecular weight of the PEG. The supramolecular nature allows for a controlled and simple incorporation of bioactivity (vide infra).

A second supramolecular unit that is used as cell-supportive and instructive material are the peptide amphiphiles (PAs) developed in the Stupp laboratory. ${ }^{[37]}$ These peptide amphiphiles contain an aliphatic hydrocarbon chain with a peptide block that contains a hydrophobic sheet-forming sequence, 1-3 charged amino acids that improve water solubility, and a bioactive signaling epitope. The self-assembled fibers yield scaffold materials that support cells and can signal cells for differentiation when bioactive cues are included (vide infra). ${ }^{[38]}$ Programmable control of properties is found in the balance of peptide versus chain sizes, peptide sequences and mixing of different PA entities into a heterogeneous fiber. Mechanical properties are reported as storage modulus in the range of 200-1000 $\mathrm{Pa},{ }^{[39]}$ charge and polarity are controlled by the nature of the amino acids.

Besides peptide amphiphiles, supramolecular ECM-mimicking materials have been self-assembled solely by peptides. Using the secondary structure of $\beta$-sheets, peptide sequences can be designed (programmed) to generate multifunctional hydrogels, displaying mechanical properties (storage modulus) of $20-123 \mathrm{kPa} \cdot{ }^{40]}$ The stiffness could be controlled through crosslinking peptides bearing orthogonal reactive groups with heterobifunctional crosslinkers. Although programmable through peptide design, this approach diverts the system from true supramolecular to mixed covalent/supramolecular.

As the base of our genetic code, DNA is a versatile material; the deoxynucleotides form double stranded-helices with features such as stability, flexibility, and precise programmability, which render them suitable for ECM-mimicking hydrogels. ${ }^{[41]}$ DNA can be the main constituent of a hydrogel, the cross-link or both. By 5 '-end functionalization with acrydite, the DNA strands have been covalently coupled to a polyacrylamide base to allow hydrogel formation. The (DNA) crosslinks are tunable per base, as every nucleotide pair can be programmed. By changing the level of cross-linking and the length of the cross-link (e.g. the DNA strand), hy- drogels with a stiffness range of 0.1-30 $\mathrm{kPa}^{[29]}$ were obtained, with the negative charge of DNA conferring a similar overall negative charge to the hydrogel. DNA as cross-link is reversibly tunable through $\mathrm{pH}$ and temperature. Due to the per-base modulation of cross-links, programmable control over this type of cross-link versus covalent cross-links is a clear benefit for property design. DNA as a material shows great potential for future engineering of functional materials. Branched forms such as Holliday junctions ${ }^{[42]}$ can be used to add complexity in the crosslink providing a new toolbox in hydrogel design.

Bulk DNA hydrogels are made from a network solely comprised of DNA strands, and reportedly exhibit a stiffness in the relevant range from $1.5-41.5 \mathrm{kPa} .{ }^{[43]}$ These hydrogels are likewise degradable through $\mathrm{pH}$ and temperature and, due to the use of DNA as the sole component, display a dominant negative charge. The programmability of such hydrogels is extraordinary as every nucleotide base can be addressed. Additional programmable control is provided by the various branching properties; $\mathrm{X}, \mathrm{Y}$ and $\mathrm{T}$ shaped junctions in the branched regions further impose control over the DNA hydrogel network. ${ }^{[41]}$

\subsection{Programmable Control in Recombinant Protein Materials}

A class of biomaterials that uses the programmable natural toolbox are proteinengineered polymers. Their tunable nature enables programmable tailoring of a range of biomaterial properties, creating an interesting alternative approach to synthetic polymers and natural scaffolds. The units that make up the polymer are derived from natural ECM proteins, but can be mix-andmatched into a new DNA plasmid, transfected into an organism of choice, and expressed and purified to yield a biopolymer with exact molecular-level sequence control. Because of the modular design strategy of protein-engineered biomaterials, these scaffolds can be easily modified to introduce and fine-tune cell-adhesion, elasticity and degradability. ${ }^{[4]}$ Through this modular design, recombinant protein hydrogels possess a great degree of inherent programmability. By interspersing degradable motifs at arbitrary sites in the protein, the recombinant hydrogels demonstrate the highest level of programmable control of all systems reviewed.

Elastin is one of the most abundant elastomeric proteins, and provides tensile strength and elasticity to tissues such as connective tissues, lungs, skin, and blood vessels. ${ }^{[45]}$ The precursor of elastin is tropoelastin, which is comprised of the hydrophobic pentapeptide repeats Val-Pro-GlyX-Gly (VPGXG, where $\mathrm{X}$ is a hydrophobic amino acid) and alanine-rich domains 
containing lysine.[46] Such pentapeptide repeats have been used as the building blocks to construct elastin-like polypeptides (ELPs), (VPGXG) ${ }_{m}$ (where $m$ typically ranges from 20 to 330 ). Following pioneering work on ELP polypeptides accomplished by Urry and co-workers, ELP-based protein hydrogels, including their structure-function relationship and potential in biomedical application, have been heavily investigated.[47] VPGXG sequences are among the best-characterized building blocks within the class of ELPbased materials, and have been widely used in hydrogel construction. ${ }^{[48]}$

Recombinant protein hydrogels with a modular design based on elastin-like sequences have been engineered by Heilshorn et al. ${ }^{[49]}$ Protein sequences with elastin-like sequences, bio-active sites and cleavage sites were expressed and purified in $E$. coli after which they are crosslinked through NHS ester and primary amine reactions. The resulting hydrogels have a range in elastic modulus of $46-63 \mathrm{kPa}$. These hydrogels were designed for neurite outgrowth, and local degradation was modulated by the presence of cleavage sites vulnerable to a protease secreted from the tips of growing neurites. Due to the recombinant protein nature, this hydrogel exhibits an inherent programmable nature where sequence control precisely defines hydrogel properties.

Materials developed in the Tirrell lab display an additional level of programmable control in properties. Next to sequence control through genetic engineering, sitespecific and/or residue-specific incorporation of unnatural amino acids was shown to allow for the tuning of the material's stiffness. The methods are complementary: residue-specific incorporation allows engineering of the overall physical and chemical behavior of proteins and proteinlike macromolecules, whereas site-specific methods allow mechanistic questions to be probed in atomistic detail.[50] Artificial extracellular matrix proteins constructed from alternating CS5 cell-binding domains (from fibronectin) and (VPGIG) $x$ domains (from elastin) were cross-linked through site-specific cross-linking with hexamethylene diisocyanate in dimethylsulfoxide. Since the primary amine functional groups were located only at the ends of the protein, amine-selective reagents should be capable of cross-linking the proteins without disrupting the cell binding domains. Elasticities in the range of 300-1000 $\mathrm{kPa}^{[51]}$ were reported, matching properties of natural elastin $(300-600 \mathrm{kPa}) .{ }^{[52]}$

Materials from similar artificial extracellular matrix proteins modified with variable amounts of the unnatural amino acid $p$-azido-Phe $\left(\mathrm{N}_{3} \mathrm{Phe}\right)$ were developed by the same group. ${ }^{[51]}$ The phenylalanine
(Phe) sites encoded within the elastin-like domains of the protein served as sites for the incorporation of the non-canonical amino acid $p$-azidophenylalanine. Ultraviolet irradiation yielded cross-linked elastomers with moduli in the range of 0.3-1.0 $\mathrm{MPa}$ that could be tuned through variation in exposure time and $\mathrm{N}_{3}$ Phe content. Mammalian cells adhered selectively to regions of the surface patterned with protein, and were absent from the other regions of the material. Although it is possible to program non-natural amino acids into recombinant proteins, challenges still exist in the production of large-scale quantities of materials through recombinant techniques.

Combinatorial recombinant protein hydrogels offer another aspect of tenability and programmable control. By combining three distinct fibril-forming proteins a more heterogeneous hydrogel can be formed. Fibril-forming proteins were initially produced in $P$. pastoris, both functionalized and non-functionalized. When mixed at high protein concentrations (more than several $\mathrm{g} / \mathrm{L}$ ), gels form due to an entanglement of long fibrils and weak physical cross-links, yielding a range of low elastic moduli of $0.5-1.5 \mathrm{kPa} .^{[53]} \mathrm{At}$ low $\mathrm{pH}$, the fibrils are soluble due to the positively charged histidines but once the charges are neutralized at neutral $\mathrm{pH}$ some domains form secondary structures like $\beta$-sheets. The system is fairly programmable due to its modular design principle and the prospect of heterogeneous hydrogels is promising (due to its closer resemblance to nature) but the lack of control over crosslinking and spatial distribution of bio-functionalization requires attention.

\subsection{Overview of Bulk Properties in ECM-mimicking Systems}

Programmability in materials properties can be achieved in both covalent and physical hydrogel systems, and generally a stiffness matching that of natural tissue can be achieved (Table 1). Adaptive yet robust behavior in covalent synthetic systems has been demonstrated through the introduction of enzyme-degradable or chemically instable cross-links. The systems discussed show a wide range in mechanical properties covering almost the full natural spectrum. However, the synthesis of very soft but stable hydrogel architectures remains a challenge, and one likely to be important for the next step in true ECM mimicking architectures.

\section{Strategies for (Controlled) Bio-functionalization}

Current work re-establishes that stiffness of a material is the defining factor for differentiation, ${ }^{[61]}$ rather than protein tethering. However, bioactive cues are necessary for selective cell adhesion, instruction, and proliferation. ${ }^{[62,63]}$ Anti-fouling polymer hydrogel systems need to be biofunctionalized to become suited for cellular support. Bio-recognition sites in natural ECM polymers are spatially well-defined and abundant; likely it is this heterogeneous composition of spatially defined active sites that makes Matrigel advantageous for tissue engineering compared to synthetic materials. Controlled biofunctionalization of synthetic hydrogels therefore will provide a means to improve ECM performance. We continue our analysis on the previously described systems for programmable ligand incorporation.

\subsection{Identities of Bio-active Ligands}

The main bio-active cues used for material functionalization have been identified and derived from natural ECM proteins, with the RGD sequence as classic motif. The RGD peptide was identified from the fibronectin cell-binding $\mathrm{III}_{10}$ and recognized by combinations of $\alpha \beta$ integrins. ${ }^{[64]}$ When the RGD motif is coated on a surface, it modulates cell adhesion through valency ${ }^{[65]}$ and distribution, ${ }^{[66]}$ showcasing the need for spatial organization of the RGD motifs. In addition, the PHSRN peptide was identified as a synergistic region for the RGD motif and both are present on fibronectin separated by a $30-40 \AA$ distance. ${ }^{[67]}$ When examining the effect of this synergistic effect on osteoblasts (using a $\sim 40 \AA \mathrm{G}_{13}$ linker as spacer), the combination of RGD and PHSRN improved adhesion, spreading, and focal contact formation versus RGD alone. ${ }^{[68]}$ Many other bioactive regions for cell adhesion have been recognized on natural proteins, the $\alpha_{4} \beta_{1}$ binding LDV and REDV ${ }^{[69]}$ motif (identified from fibronectin), the $\alpha_{M} \beta_{2}$ binding KRLDGS motif (identified from fibrinogen), the $\alpha_{2} \beta_{1}$ binding by YGYYGDALR and FYFDLR (identified from laminin and type IV collagen respectively). Despite these other candidates, RGD has obtained the central role in adhesion biology by 1) being the earliest discovery of a small binding motif that binds integrins and 2) being able to bind the largest number of different integrins as compared to other binding motifs (about $40 \%$ of the now 24 established integrins).[64] Recreating the synergistic effect of RGD with PHSRN had proven advantageous, but leaves out many of the other instructing cues found in the natural ECM and Matrigel. To close the gap in performance of synthetic materials versus Matrigel, the introduction of additional motifs with correct spatial organization should imitate the natural ECM more accurately. 
Table 1. Summary of material properties found in each type of reviewed ECM mimicking hydrogel system (covalent, supramolecular, recombinant and natural) analyzing critical properties: stiffness, robustness, anti-fouling, charge and control over bio-functionalization. The formulation of functional synthetic ECMs is compared with parameters of the natural ECM, guiding future ECM research. (Stiffness is defined in the Young's modulus (E) or the storage modulus $\left(\mathrm{E}_{\mathrm{s}}\right.$ only for PA: peptide amphiphiles))

\begin{tabular}{|c|c|c|c|c|}
\hline Property & Covalent & Supramolecular & Recombinant & Natural $^{\mathbf{a}}$ \\
\hline Stiffness (range) & $\begin{array}{l}\mathrm{E}_{\text {Void }}: 5-110 \mathrm{kPa}^{[21]} \\
\mathrm{E}_{\text {photo }}: 10 \rightarrow 2 \mathrm{kPa}^{[9]} \\
\mathrm{E}_{\text {Lutolf }}: 0.3-1.7 \mathrm{kPa}^{[19]} \\
\mathrm{E}_{\mathrm{HA}}: 2.5-30 \mathrm{kPa}^{[23]} \\
\mathrm{E}_{\text {HA-MMP }}: 4.4-91.1 \mathrm{kPa}^{[24]} \\
\mathrm{E}_{\text {pNIPAAm }}: 0.5-10 \mathrm{kPa}^{[25]}\end{array}$ & $\begin{array}{l}\mathrm{E}_{\mathrm{UPy}}: 49 \mathrm{MPa}^{[30]} \\
\mathrm{E}_{\mathrm{DNAcrosslink}}: 0.1-30 \\
\mathrm{kPa}^{[29]} \\
\mathrm{E}_{\mathrm{DNApure}}: 1.5-41.5 \\
\mathrm{kPa}^{[33]} \\
\mathrm{E}_{\text {storage PA }}: 200-1000 \\
\mathrm{kPa}^{[39]}\end{array}$ & $\begin{array}{l}\mathrm{E}_{\text {elastin }}: 1.5-910 \mathrm{kPa}^{[47]} \\
\mathrm{E}_{\text {Heilshorn }}: 46-63 \mathrm{kPa}^{[49]} \\
\mathrm{E}_{\text {Tirrel }}: 0.3-1.0 \mathrm{MPa}^{[51]} \\
\mathrm{E}_{\text {nanofibrilar }}: 0.5-1.5 \\
\mathrm{kPa}^{[53]}\end{array}$ & $\begin{array}{l}\mathrm{E}_{\text {neuron }}: 0.05-0.3 \\
\mathrm{kPa}^{[14]} \\
\mathrm{E}_{\text {muscle }}: 12 \mathrm{kPa}^{[15]} \\
\mathrm{E}_{\text {bone }}: 20-110 \mathrm{kPa}^{[16]}\end{array}$ \\
\hline Robustness & $\begin{array}{l}\text { Photodegradation }^{[9]} \\
\text { MMP sensitive motifs }{ }^{[24]} \\
\text { Oxidation }^{[21]}\end{array}$ & $\begin{array}{l}\text { Temperature }^{[28]} \\
\text { pH sensitive } \\
\text { regions }^{[27]}\end{array}$ & $\begin{array}{l}\text { Enzyme degradable } \\
\text { motifs }^{[54]}\end{array}$ & $\begin{array}{l}\text { MMP mediated } \\
\text { degradation }^{[55] \mathrm{c}}\end{array}$ \\
\hline Anti-fouling ${ }^{\mathrm{b}} \&$ charge & $\begin{array}{l}\text { Mainly anti-fouling } \\
\text { Inert }^{[56]} / \\
\text { charged }^{[57]}\end{array}$ & $\begin{array}{l}\text { Mainly anti-fouling } \\
\text { Inert }{ }^{[56] /} \\
\text { charged }^{[57]}\end{array}$ & $\begin{array}{l}\text { Fouling } \\
\text { Variable }^{[58]}\end{array}$ & $\begin{array}{l}\text { Fouling }^{[59]} \\
\text { Variable }^{[6,60]}\end{array}$ \\
\hline $\begin{array}{l}\text { Controlled bio- } \\
\text { functionalization }\end{array}$ & $\begin{array}{l}\text { Control over density, but } \\
\text { not spatial. Not dynamic }\end{array}$ & $\begin{array}{l}\text { Control over } \\
\text { density but not } \\
\text { spacing, dynamic } \\
\text { redistribution }\end{array}$ & $\begin{array}{l}\text { Spatial control } \\
\text { through genetic } \\
\text { engineering }\end{array}$ & $\begin{array}{l}\text { Naturally defined } \\
\text { spacing. }\end{array}$ \\
\hline
\end{tabular}

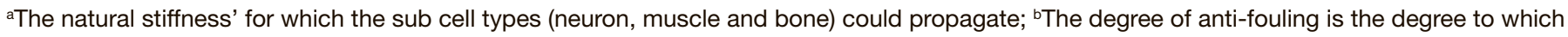
the material retains morphogens and adheres to non-specific targets; 'In addition to MMP degradation there are many other proteolytic enzymes remodeling the natural ECM.

\subsection{Covalent versus Supra- molecular versus Integrated Ligand Presentation}

Bio-functionalization can be either covalent, through supramolecular interactions or integrated in the recombinant protein domains (Fig. 2). Covalent bio-functionalization limits the dynamic relocation of bioactive cues on the material, however, the presentation is very stable. When included in monomer-design, spacing can be varied by the polymerization mechanism and introduction of block-copolymers, ${ }^{21]}$ where one block is functionalized, and the other not (or orthogonally presenting a different signal). Although spacing can be varied to some extent, functional programmable control over spacing and heterogeneous presentation of bioactive ligands through covalent synthesis has not been achieved to date.

Bio-functionalization in covalent systems is generally achieved through classic conjugation chemistries. Examples found in the hydrogel systems discussed in section 2 include amide bond formation (void and phototunable hydrogel), maleimide chemistry using thiol-functionalized RGD groups (hyaluronic acid gels) and endfunctionalized transglutaminase coupled with PEG (Lutolf's synthetic matrix).
Control over ligand density and spacing in these systems is limited to the availability of active sites in the polymer and cannot (fully) be programmed without the loss of bulk material properties. For pNIPAAmbased architectures, functionalization is achieved through co-polymerization or mixing with different polymers that allow for direct functionalization, limiting this system in its programmable control over ligand spacing ${ }^{[70]}$ (Table 1).

While many biological systems excel at controlling supramolecular polymerization, synthetic chemistry provides a library of functional groups not found in nature. Synthetic supramolecular polymers are able to combine the best of two worlds: bioinspired functionality from natural systems and the accessibility and features of synthetic compounds. In DNA-based materials, this is possible through the choice of nucleotides (A-T/C-G, with 2 or 3 hydrogen bonds respectively). For UPy-based networks, bioactive cues can be introduced using UPy/diUPy functionalization (with 4 and $2 \times 4$ hydrogen bonds respectively). In the peptide amphiphile system, the bioactive peptides are abundantly present and are integrated in the fiber self-assembly providing the necessary bioactive cues yet are spatially uncontrolled. ${ }^{40]}$ For the $\beta$-sheet peptide hydrogels, RGD-extensions on the peptide-aggregating domains were included and the growth of cells could be modulated. However, the precise spacing of the functional elements on the fibers remained unknown and uncontrolled. Supramolecular bio-functionalization is inherently dynamic, and the strength between the ligand and material backbone can be tuned. However, an inherent challenge for bio-functionalization in dynamic supramolecular systems is the dissociation of ligands leading to loss in functionality.

Recombinant protein hydrogels are inherently bio-functional through coding the presence of bioactive regions in the protein sequence. Additional variation in ligand density has been accomplished via mixing of different artificial ECM proteins, displaying RGD domains and inactive RDG domains. ${ }^{[63]}$ Increasing the density of RGD domains in cross-linked films resulted in more robust cell adhesion and spreading but did not affect cell migration. Control of cell-binding domain density in artificial ECM proteins can thus be used to modulate cell-material interactions and serves as an important design tool for control in ECM mimicking materials. 


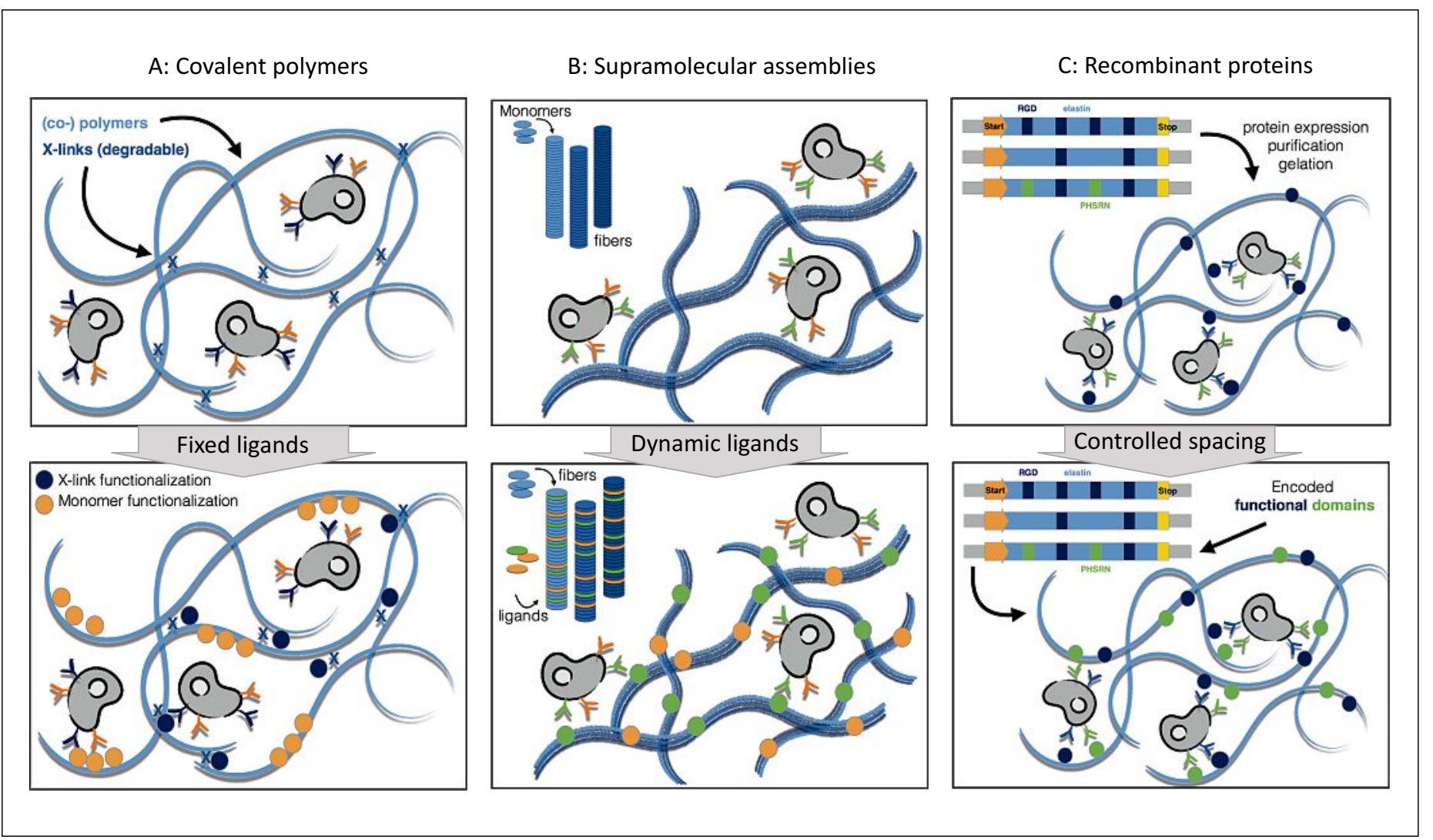

Fig. 2. (A; Top) Covalent polymers form a static hydrogel with (potential) degradable cross links (indicated by blue $X$ ), when bio-functionalized (A; Bottom) ligands are present at fixed locations and biological mismatches occur, resulting in suboptimal performance of the system as ECM mimic. (B; Top) Supramolecular assemblies form hydrogels with dynamic interactions between the fibrous monomers, when bio-functionalized (B: Bottom) the dynamic presentation of ligands allow for adjustable cell-material interactions, however spacing cannot be truly programmed and not all ligands will be effective for cell-adhesion. (C; Top) Recombinant proteins have the position of crosslinks and bio-functionalization engineered into the modular sequences which allows (C; Bottom) for accurate spacing of cell-adhesion sites.

\section{Future Thoughts on Programmable Control in ECM- mimicking Materials}

Structure and function, material and cells, one shapes the other and vice-versa. This natural dynamic reciprocity shapes developing cells into defined niches and phenotypes. It is a concept of exchange of information between cells and their environment in a programmable fashion. Without programmability, dynamic reciprocity is not achievable. When only oneway traffic occurs, cell and material are not optimally interacting, and the synthetic hydrogel is not a true ECM mimic. Many recent advances show improvements in the material-cell interplay through the introduction of degradability and functionalization. Dynamic crosslinks allow for simple reciprocity and bio functionalization provides instructive signals to cells. However, to date not one system achieves a display of the full programmable ensemble of properties found in nature.

The challenge for further research is to progress from degradable cross-links in the matrix towards dynamic reciprocal interactions. Both covalent and supramolecular bio-functionalization have the same prospect of spatial control but the latter offers the introduction of dynamicity. While these dynamics can result in reciprocal interactions, they also lead to unstable functionalization, which makes control over the cell-material interplay very challenging. In principle, engineered artificial proteins could display any combination of functions found in natural proteins including catalysis, binding, signaling, and transport. The ability to hybridize multiple functions in a single protein through genetic engineering and the possibility to carefully control the spacing between active units is an advantage over covalent and supramolecular approaches that require chemical modifications to make materials biocompatible or bioactive. The programmable spatial control of bioactive sites in these systems is promising but commercial translation remains limited by expression yields of these systems and purification from host expression systems leading to immunogenic reactions.

Current bio-functionalization strategies are predominantly focused on the incorporation of RGD and its density and spacing. However, many more bioactive peptide sequences have been reported to assist in cell-adhesion and instruction. ${ }^{[64]}$ The exact spacing between RGD and the synergistic PHSRN is known (30-40 ̊). However, due to the lack of spatial control in ligand presentation, hydrogels that preserve and present this spacing are non-existent. With an array of active cues present in nature, limiting ourselves to one or two peptides might be too minimal. Perhaps our limited success in the design of true ECM mimicking hydrogels can be explained along these lines.

Implementation of a programmable dynamic reciprocal relationship between the material-cell interplay is needed for true ECM-mimicking hydrogels. Complex combinations of several ECM constituents with functionalized PEG, as shown by Lutolf et al., ${ }^{[19]}$ present a remarkable functional matrix. Further advances should focus on the introduction of programmable spatial and dynamic control in ligand presentation and copying this complexity synthetically. The ideal system should combine the dynamics found in supramolecular materials, with the robustness from covalent and the bioactive properties from recombinant ECMs. Only when we discover how to combine all properties in a programmable reciprocal fashion, will true artificial ECM materials be achieved. 
[1] R. O. Hynes, Science 2009, 326, 1216.

[2] B. G. Keselowsky, D. M. Collard, A. J. García, J. Biomed. Mater. Res. A 2003, 66, 247.

[3] R. J. Miron, M. Fujioka-Kobayashi, D. Buser, Y. Zhang, D. D. Bosshardt, A. Sculean, Int. J. Oral Maxillofac. Implants 2017, 32, 196.

[4] K. J. Warren, D. Iwami, D. G. Harris, J. S. Bromberg, B. E. Burrell, J. Clin. Invest. 2014, 124, 2204.

[5] S. Johansson, G. Svineng, K. Wennerberg, A. Armulik, L. Lohikangas, Front. Biosci. 1997, 2, 126.

[6] M. F. Brizzi, G. Tarone, P. Defilippi, Curr. Opin. Cell Biol. 2012, 24, 645.

[7] K. Shapira-Schweitzer, D. Seliktar, Acta Biomater. 2007, 3, 33.

[8] D. Missirlis, J. P. Spatz, Biomacromolecules 2014, 15, 195.

[9] C. Yang, M. W. Tibbitt, L. Basta, K. S. Anseth, Nat. Mater. 2014, 13, 645.

[10] W. J. Kao, D. Lee, Biomaterials 2001, 22, 2901

[11] H. K. Kleinman, G. R. Martin, 'Matrigel: basement membrane matrix with biological activity', in 'Seminars in cancer biology', Elsevier; 2005, pp 378-86

[12] B. K. Mann, A. S. Gobin, A. T. Tsai, R. H. Schmedlen, J. L. West, Biomaterials 2001, 2, 3045.

[13] J. Swift, I. L. Ivanovska, A. Buxboim, T. Harada, P. D. P. Dingal, J. Pinter, J. D. Pajerowski, K. R. Spinler, J. W. Shin, M. Tewari, F. Rehfeldt, D. W. Speicher, D. E. Discher, Science 2013, 341, 1240104.

[14] L. A. Flanagan, Y.-E. Ju, B. Marg, M. Osterfield, P. A. Janmey, Neuroreport. 2002 13, 2411.

[15] A. J. Engler, M. A. Griffin, S. Sen, C. G. Bönnemann, H. L. Sweeney, D. E. Discher, $J$. Cell Biol. 2004, 166, 877.

[16] H. J. Kong, T. R. Polte, E. Alsberg, D. J. Mooney, Proc. Natl. Acad. Sci. USA 2005, 102, 4300.

[17] M. Larsen, V. V. Artym, J. A. Green, K. M. Yamada, Curr. Opin. Cell Biol. 2006, 18, 463.

[18] D. S. Benoit, M. P. Schwartz, A. R. Durney, K. S. Anseth, Nat. Mater. 2008, 7, 816

[19] N. Gjorevski, N. Sachs, A. Manfrin, S. Giger, M. E. Bragina, P. Ordóñez-Morán, H. Clevers, M. P. Lutolf, Nature 2016, 539, 560.

[20] J. A. Killion, S. Kehoe, L. M. Geever, D. M. Devine, E. Sheehan, D. Boyd, C. L. Higginbotham, Mater. Sci. Eng. C 2013, 33, 4203.

[21] N. Huebsch, E. Lippens, K. Lee, M. Mehta, S. T. Koshy, M. C. Darnell, R. M. Desai, C. M. Madl, M. Xu, X. Zhao, O. Chaudhuri, C. Verbeke, W. S. Kim, K. Alim, A. Mammoto, D. E. Ingber, G. N. Duda, D. J. Mooney, Nat. Mater. 2015, 14, 1269.

[22] X. Z. Shu, Y. Liu, F. Palumbo, G. D. Prestwich, Biomaterials 2003, 24, 3825.

[23] S. Khetan, J. A. Burdick, Biomaterials 2010 , 31,8228 .
[24] S. Khetan, M. Guvendiren, W. R. Legant, D. M. Cohen, C. S. Chen, J. A. Burdick, Nat. Mater 2013, 12, 458

[25] S. Chen, J. Shi, X. Xu, J. Ding, W. Zhong, L. Zhang, Colloids Surf B Biointerfaces 2016, 140, 574

[26] Mebiol ${ }^{\circledR}$-Thermoreversible hydrogel - Catalog \#5180-10mL. Available from: https://www.ad vancedbiomatrix.com/thermoreversible-hydrogel/mebiol-lyophilized-10-ml-5180-10ml/

[27] W. Guo, C.-H. Lu, R. Orbach, F. Wang, X.-J. Qi, A. Cecconello, D. Seliktar, I. Willner, $A d v$. Mater. 2015, 27, 73.

[28] Y. Xing, E. Cheng, Y. Yang, P. Chen, T. Zhang, Y. Sun, Z. Yang, D. Liu, Adv. Mater. 2011, 23, 1117.

[29] F. X. Jiang, B. Yurke, B. L. Firestein, N. A. Langrana, Ann. Biomed. Eng. 2008, 36, 1565.

[30] P. Y. Dankers, M. C. Harmsen, L. A. Brouwer, M. J. Van Luyn, E. W. Meijer, Nat. Mater. 2005, 4, 568.

[31] T. F. De Greef, M. M. Smulders, M. Wolffs, A. P. Schenning, R. P. Sijbesma, E. W. Meijer, Chem. Rev. 2009, 109, 5687.

[32] B. A. Schweitzer, E. T. Kool, J. Am. Chem. Soc. 1995, 117, 1863

[33] J. K. Hirschberg, L. Brunsveld, A. Ramzi, J. A. Vekemans, R. P. Sijbesma, E. W. Meijer, Nature 2000, 407, 167.

[34] R. P. Sijbesma, F. H. Beijer, L. Brunsveld, B. J. Folmer, J. K. Hirschberg, R. F. Lange, J. K. Lowe, E. W. Meijer, Science 1997, 278, 1601.

[35] P. Y. Dankers, T. M. Hermans, T. W. Baughman, Y. Kamikawa, R. E. Kieltyka, M. Bastings, H. M. Janssen, N. A. Sommerdijk, A. Larsen, M. J. van Luyn, A. W. Bosman, E. R: Popa G. Fytas, E. W. Meijer, Adv. Mater. 2012, 24 , 2703.

[36] A. C. H. Pape, M. Bastings, R. E. Kieltyka, H. M. Wyss, I. K. Voets, E. W. Meijer, P. Y. W. Dankers, Int. J. Mol. Sci. 2014, 15, 1096.

[37] H. Cui, M. J. Webber, S. I. Stupp, Pept. Sci. 2010, $94,1$.

[38] G. A. Silva, C. Czeisler, K. L. Niece, E. Beniash, D. A. Harrington, J. A. Kessler, S. I. Stupp, Science 2004, 303, 1352.

[39] M. O. Guler, L. Hsu, S. Soukasene, D. A. Harrington, J. F. Hulvat, S. I. Stupp, Biomacromolecules 2006, 7, 1855

[40] J. Z. Gasiorowski, J. H. Collier, Biomacromolecules 2011, 12, 3549 .

[41] J. Li, L. Mo, C.-H. Lu, T. Fu, H.-H. Yang, W. Tan, Chem. Soc. Rev. 2016, 45, 1410

[42] N. C. Seeman, Nature 2003, 421, 427.

[43] S. H. Um, J. B. Lee, N. Park, S. Y. Kwon, C. C. Umbach, D. Luo, Nat. Mater. 2006, 5, 797.

[44] D. Sengupta, S. C. Heilshorn, Tissue Eng. Part B Rev. 2010, 16, 285.

[45] Z. Indik, H. Yeh, N. Ornstein-Goldstein, P. Sheppard, N. Anderson, J. C. Rosenbloom, L. Peltonen, J. Rosenbloom, Proc. Natl. Acad. Sci. USA 1987, 84, 5680.
[46] D. W. Urry, T. Hugel, M. Seitz, H. E. Gaub, L. Sheiba, J. Dea, J. Xu, T. Parker, Philos. Trans. R. Soc. Lond. B Biol. Sci. 2002, 357, 169.

[47] N. Annabi, S. M. Mithieux, G. Camci-Unal, M R. Dokmeci, A. S. Weiss, A. Khademhosseini, Biochem. Eng. J. 2013, 77, 110.

[48] M. R. Banki, F. Liang, D. W. Wood, Nat. Methods 2005, 2, 659.

[49] K. S. Straley, S. C. Heilshorn, Soft Matter 2009 , 5,114 .

[50] A. J. Link, M. L. Mock, D. A. Tirrell, Curr. Opin. Biotechnol. 2003, 14, 603.

[51] P. J. Nowatzki, C. Franck, S. A. Maskarinec, G Ravichandran, D. A. Tirrell, Macromolecules 2008, 41,1839 .

[52] P. J. Nowatzki, D. A. Tirrell, Biomaterials 2004 25,1261

[53] M. K. Włodarczyk-Biegun, M. W. Werten, U. Posadowska, I. M. Storm, F. A. de Wolf, J. J. van den Beucken, S. C. Leeuwenburgh, M. A. Cohen Stuart, M. Kamperman, J. Biomed. Mater. Res. A 2016, 104, 3082.

[54] K. S. Straley, S. C. Heilshorn, Adv. Mater. 2009, $21,4148$.

[55] Z. Werb, Cell 1997, 91, 439.

[56] C.-C. Lin, K. S. Anseth, Pharm. Res. 2009, 26, 631.

[57] F. Tan, X. Xu, T. Deng, M. Yin, X. Zhang, J. Wang, Biomed. Mater. 2012, 7, 55009.

[58] S. C. Rizzi, J. A. Hubbell, Biomacromolecules 2005, 6, 1226.

[59] M. Strigini, J. Neurobiol. 2005, 64, 324.

[60] A. W. Palmer, R. E. Guldberg, M. E. Levenston, Proc. Natl. Acad. Sci. USA 2006, 103, 19255.

[61] J. H. Wen, L. G. Vincent, A. Fuhrmann, Y. S Choi, K. C. Hribar, H. Taylor-Weiner, S. Chen, A. J. Engler, Nat. Mater. 2014, 13, 979.

[62] D. Grafahrend, J. L. Calvet, J. Salber, P. D. Dalton, M. Moeller, D. Klee, J. Mater. Sci. Mater. Med. 2008, 19, 1479.

[63] J. C. Liu, D. A. Tirrell, Biomacromolecules 2008, 9, 2984.

[64] E. Ruoslahti, Annu. Rev. Cell. Dev. Biol. 1996, 12, 697.

[65] X. Montet, M. Funovics, K. Montet-Abou, R. Weissleder, L. Josephson, J. Med. Chem. 2006, 49, 6087.

[66] G. Maheshwari, G. Brown, D. A. Lauffenburger, A. Wells, L. G. Griffith, J. Cell Sci. 2000, 113, 1677.

[67] D. J. Leahy, I. Aukhil, H. P. Erickson, Cell 1996, $84,155$.

[68] D. S. Benoit, K. S. Anseth, Biomaterials 2005, 26, 5209.

[69] M. Leiss, K. Beckmann, A. Girós, M. Costell, R. Fässler, Curr. Opin. Cell Biol. 2008, 20, 502.

[70] M. Ebara, Y. Kotsuchibashi, R. Narain, N. Idota, Y.-J. Kim, J. M. Hoffman, K. Uto, T. Aoyagi, 'Smart Biomaterials', Springer, 2014. 